\title{
The induced asynchrony effect: Its role in visual judgments of temporal order and its relation to other dynamic perceptual phenomena
}

\author{
C. E. COLLYER \\ Princeton University, Princeton, New Jersey 08540
}

\begin{abstract}
A context-induced "illusion" in visual judgments of temporal order, termed the induced asynchrony effect (IAE), is reported. It consists of an apparent ordering in time of two simultaneous light onsets, produced by the preceding, asynchronous offsets of two other lights. The joint effect of a real stimulus onset asynchrony and a preceding stimulus offset asynchrony on judgments of onset order appears to be additive, given a Gaussian transformation of response probability. This result is shown to be consistent with a simple statistical decision model, which provides a conceptual framework for drawing inferences from temporal order judgment data. However, it is emphasized that certain interpretations of such models are not empirically testable on the basis of temporal order data alone. An attempt is made to relate the IAE to three other dynamic perceptual phenomena; all four effects may reflect a tendency of observers to perceive the velocity of apparent motion as being constant. Questions raised by the demonstration of the IAE are discussed, and directions for further research are suggested.
\end{abstract}

In the course of designing stimulus displays for use in experiments on apparent motion, temporal order, and masking, a robust "illusion" in visual judgments of temporal order was encountered. Briefly, if two stimuli are successively extinguished in the spatial vicinity of two subsequent "test" stimuli, the apparent onset order of the test stimuli is strongly influenced by the offset order of the two preceding stimuli. More specifically, the onset of that test stimulus nearest the initially extinguished stimulus, tends to be judged earlier than the other test stimulus onset. When the two test onsets are really simultaneous, the offset asynchrony of the two preceding stimuli can be sufficient to induce a perception of onset ordering. This "illusion" is referred to as the induced asynchrony effect (IAE).

Two experiments are reported in this paper; the first demonstrates the reliability of the IAE, while the second examines the joint influence of two factors on temporal order judgments (TOJs): test stimulus onset asynchrony and preceding stimulus offset asynchrony.

\section{EXPERIMENT I}

This experiment was designed to demonstrate the robust and reliable nature of the IAE using an optimal "inducing" procedure. A questionnaire was employed to allow a number of observers to characterize the apparent "onset order" of two lights which were actually illuminated simultaneously in proximity to two previously extinguished "inducing"

The author wishes to thank R. A. Kinchla for many valuable discussions and suggestions concerning the present study in particular and relations between theory and data in general. The author's address is: Department of Psychology, Princeton University, Princeton, New Jersey 08540. lights. Three experimental conditions were defined which differed only in the offset sequence of these inducing lights: Sequence $L$, in which the leftmost inducing light was extinguished first; Sequence $R$, in which the right light was extinguished first; and Sequence $S$, in which the inducing lights were extinguished simultaneously.

\section{Method}

Subjects. Twenty unpaid students and colleagues served as observers.

Apparatus and Stimuli. Stimulus displays were presented on a Tektronix Type 602 CRT display unit, controlled by a PDP-12 computer (Digital Equipment Corporation). The spatial configuration of the stimulus display and an example of the time course of one trial are shown in Figure 1. The stimuli were illuminated rectangular patches consisting of a 6 by 4 matrix of closely spaced points.

Three display programs were prepared, each of which presented a repeating series of identical trials in one of the three previously indicated sequences. In Sequence $L$, the time course of stimulus events within a trial was as shown in Figure $1 \mathrm{~b}$; that is, the offset of Stimulus A occurred $100 \mathrm{msec}$ before the offset of Stimulus B, followed 50 msec later by the simultaneous onsets of Stimuli $C$ and D. Note that 150 msec is usually considered too brief to permit an overt fixation shift to be initiated and completed (Komoda, Festinger, Phillips, Duckman, \& Young, 1973). In Sequence R, the offset of Stimulus B occurred $100 \mathrm{msec}$ before the offset of Stimulus A; the durations of Stimuli and A and B were 1,100 and $1.000 \mathrm{msec}$, respectively. In Sequence $S$, the offsets of Stimuli A and $B$ were simultaneous; the durations were both $1.000 \mathrm{msec}$. In all three sequences, the onsets of $C$ and $D$ were simultaneous; a brief 50 -msec dark interval elapsed between the last offset (of $A$ or $B$ ) and the simultaneous onsets (of C and D). An interval of $2,000 \mathrm{msec}$ elapsed between successive presentations of the identical stimulus patterns in each of the three conditions.

In addition to the three IAE sequences, a program was prepared which controlled the display of a stimulus pair, in which the onset order of the stimuli could be selected by the experimenter. Using a Teletype keyboard to initiate single trials, the experimenter could present the two stimuli, in a "right first" (by $50 \mathrm{msec}$ ), "left first" 


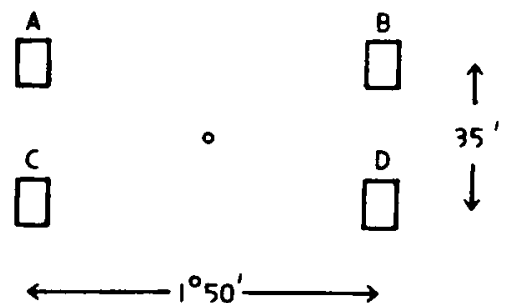

(a)

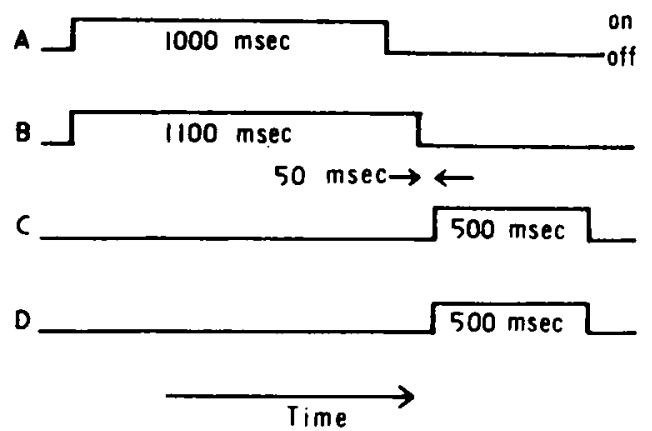

(b)

Figure 1. (a) Spatial configuration of stimulus position in the IAE display of Experiment I. (b) Time coune of ylumination of stimull in the IAE display of Experiment I, for Sequence $L$.

(by $50 \mathrm{msec}$ ), or "simultaneous" order. The two stimuli were laterally separated by $1^{\circ} 50^{\prime}$ visual angle at a viewing distance of 30 in.; a fixation point was positioned midway between them, and remained illuminated throughout the trial. The onset of the fixation point preceded the onset of the first stimulus by $1,000 \mathrm{msec}$; the duration of the stimulus illuminated second was $500 \mathrm{msec}$.

Procedure. Subjects were run individually. All subjects weire instructed that the experimenter was interested in verifying the reliability of observers' temporal order impressions, using stimulus patterns that would be employed in a subsequent experiment. Examples of "right first," "left first," and "simultaneous" stimulus pairs were first shown to the subject, to acquaint him with the magnitude of "asynchronies" being used as well as with what the experimenter meant by "simultaneous." Then the experimenter presented a random series of nine stimulus pairs, three of each type, asking the subject to respond "right first," "left first," or "simultaneous" to identify each pair. A performance criterion of at least eight correct responses was required on this pretest series in order for an observer to be included in the final IAE tests, although subjects were not informed of this contingency. (Only one subjects, who made three errors, was rejected in this way.)

The 20 subjects who satisfied the pretest criterion, were divided into two groups. Those in Group I were shown Sequences $L$ and $S$; those in Group II were shown Sequences $R$ and $S$. Half of the subjects in each group were shown Sequence $S$ first, and half second. Subjects were given the following printed instructions prior to observing each sequence: "On the screen before you, there is a repeating display consisting of a fixation point and four stimulus lights in the following configuration: (A diagram similar to Figure $1 \mathrm{a}$ was on the instruction sheet.) Observe this display for as many repetitions as you wish. In a future experiment, observers will be asked to judge the onder in which lights $\mathrm{C}$ and $\mathrm{D}$ come on. What do you think their response tendency will be? (Please choose one of the following 3 alternatives): (i) Observers will have a strong impression that $C$ came on first; (ii) Observers will have a strong impression that $\mathrm{D}$ came on first; (iii) Observers will have the impression that $C$ and $D$ came on at about the same time."

It was hoped that by making the subject's response a "prediction" of future observers' responses, he would be more likely to give his own "immediate perceptual impression" rather than some logically deduced hypothesis about what the experimenter might really be presenting.

\section{Results}

The choices made by the 20 observers are shown in Table 1. Each row of Table 1 is a frequency distribution over the three response alternatives given a particular sequence (L, $R$, or $S)$, order of presentation (Sequence $S$ presented first or second), and group (I, which judged Sequences $S$ and L, or II, which judged Sequences $S$ and $R$ ).

All 20 subjects judged that Sequence $S$ gave an impression of onset "simultaneity." Of the 10 subjects in Group I, 9 judged that Sequence $L$ gave "a strong impression" of "left first" onset ordering. of the 10 subjects in Group II, 7 judged that Sequence $R$ gave "a strong impression" of "right first" onset ordering.

Recalling that the two test onsets in all three sequences were actually simultaneous, these data indicate considerable control of the onset order judgment by the offset asynchrony of the two preceding stimuli.

\section{THE INTERPRETATION OF TEMPORAL ORDER JUDGMENTS}

Having demonstrated that the offset asynchrony of preceding contextual stimuli is a factor influencing visual judgments of onset order, it seems natural to examine how this factor combines with real onset asynchrony to determine such judgments. This will be the purpose of Experiment II.

Table 1

Distributions of Choices: Experiment I

\begin{tabular}{cccc}
\hline & \multicolumn{3}{c}{ Choice } \\
\cline { 2 - 4 } & $\begin{array}{c}\text { "Left } \\
\text { First" }\end{array}$ & $\begin{array}{c}\text { Simul- } \\
\text { taneous }\end{array}$ & $\begin{array}{c}\text { "Right } \\
\text { First" }\end{array}$ \\
\hline $\begin{array}{c}\text { Group I } \\
\text { Sequence S }\end{array}$ & & & \\
S,L Order & 0 & 5 & 0 \\
L,S Order & 0 & 5 & 0 \\
Sequence L & & & \\
S,L Order & 5 & 0 & 0 \\
L,S Order & 4 & 0 & 1 \\
Group II & & & \\
Sequence S & & & \\
S,R Order & 0 & 5 & 0 \\
R,S Order & 0 & 5 & 0 \\
Sequence R & & & \\
S,R Order & 1 & 0 & 4 \\
R,S Order & 1 & 1 & \\
\hline
\end{tabular}


First, however, it will be useful to develop a simple model for temporal order judgments, which formalizes an interpretation of how observers convert "real" stimulus asynchronies into responses. The model will facilitate the discussion of Experiment II.

Generally speaking, the model characterizes the perceptual process as a "statistical decision" based on a "noisy" internal representation of the real onset asynchrony. Thus, it includes a measure of the hypothetical "noise level," and distinguished between the observer's response bias and sensitivity.

Formally, the model is specified as follows: Let

$$
X=A^{\prime}+P-C
$$

where $A^{\prime}$ (the "effect" of onset asynchrony), $P$ (perceptual error), and C (decision or "criterion" error) are mutually independent random variables with expected values $\mathrm{u}_{\mathrm{A}}, \mathrm{u}_{\mathrm{P}}$, and $\mathrm{u}_{\mathrm{C}}$, respectively. Let the random variable $X$ be Gaussian, with variance $\sigma_{\mathrm{X}}^{2}$, and let $\mathrm{u}_{\mathrm{A}}=\mathrm{A}$, the onset asynchrony. Let the discrete variable $R$ (the response) equal 1 if and only if

$$
\mathrm{X}<0 \text {, }
$$

and let $R$ equal 2 otherwise.

This model applies to temporal order tasks in which $A$, the onset asynchrony of two test stimuli, is an independent variable, and in which the observer's two response alternatives are "Stimulus 1 first" $\left(R_{1}\right)$ and "Stimulus 2 first" $\left(R_{2}\right)$.

This model predicts that the psychometric function (that is, the proportion of $R_{2}$ responses as a function of $A)$ will be a normal ogive. The value of that normal deviate exceeded with probability $P\left(R_{2} \mid A\right)$ will be

$$
Z\left(R_{2} \mid A\right)=-\frac{1}{\sigma}\left(A+u_{P}-u_{C}\right)
$$

that is, a linear function of $\mathrm{A}$. This linear function has a slope of $-(1 / \sigma)$, and assumes a value of zero when the onset asynchrony is

$$
a_{m}=u_{C}-u_{P} .
$$

Since the parameters $\sigma_{X}$ and $a_{m}$ are independent, the model is one which characterizes the slope and the location (or A intercept) of the psychometric function as separate aspects of performance.

Experimental manipulations other than varying $A$, may influence the slope or the location of the psychometric function, or both. Changes in slope are attributed in the model to changes in $\sigma_{\mathrm{X}}$, that is, in the total "noise" level. Changes in the location of the function may be due either to changes in response bias $\left(u_{C}\right)$ or to changes in the mean perceptual error $\left(u_{p}\right)$, or to both. Only $a_{m}$, whose value depends on both, can be estimated from TOJ data.
This inseparability of the two different sources of error has not often been emphasized, although models similar to the present one have been employed in analyzing temporal order data. Baron's (1969) "noise theory" is similar in many respects to the present model, except for his contention that "failures to correctly discriminate the order of two stimuli" are due solely to a type of perceptual error. However, if one carefully considers his use of a rating response in the temporal order task, and his interpretation of ratings as reflecting multiple criteria, it becomes clear that the value of $u_{C}$ must also be responsible for failures to discriminate order correctly.

Rutschmann's (1966) analysis of the effect of observers' overt orientation on TOJs employed a version of the present model in which $u_{C}$ was implicitly assumed to equal zero. With $a_{m}$ then theoretically equal to $u_{p}, \dot{a}_{m}$ was interpreted as the mean difference in "arrival latency" between the fixated and peripheral test stimuli. While it is very plausible to interpret $u_{P}$ as the mean difference in arrival latency (or in "transmission times"), there could be additional components of the variable $P$ whose means are nonzero. Hence, using $\hat{\mathrm{a}}_{\mathrm{m}}$ as a measure of latency difference depends on the two assumptions (i) that there are no such other components of $P$, and (ii) that $u_{C}=0$.

The use of converging evidence, such as the reaction time data of Gibbon and Rutschmann (1969), in conjunction with temporal order data seems a desirable practice in attempting to infer the role of arrival latencies in temporal order judgments. As for response bias, Gibbon and Rutschmann attributed location discrepancies between their observed psychometric functions and those predicted from RT data, to biased response criteria, which is plausible. Strictly speaking, however, the discrepancies could have arisen from sources of perceptual error (components of $\mathrm{P}$ ) to which the RT analysis was insensitive.

In short, the inseparability of $u_{P}$ and $u_{C}$ poses a problem for the interpretation of temporal order data. $A$ related problem is that the total noise, $\sigma_{\mathrm{X}}^{2}$, cannot be attributed arbitrarily to only one component of $X$, such as the perceptual error. One purpose of this paper is to draw attention to these constraints on permissible inference.

If the variable $P$ is interpreted as the difference in arrival latencies between stimuli, then the present model may be viewed as a special case of the approach summarized by Sternberg and Knoll (1973). Their representation of the psychometric function as the convolution of a decision function with an arrival latency difference distribution leads to the recognition of problems similar to those posed by the additivity of the means and variances of $A, P$, and $C$ in the present development of the model (cf. section II-D of their paper). The present model is an important special case for three reasons: (i) It makes the generally 
acceptable assumption that total error is Gaussian distributed; (ii) It employes the "deterministic" decision rule, which is the simplest of the rules considered by Sternberg and Knoll; (iii) it provides a simple, but mathematically explicit, rationale for a method of data representation (i.e., treating the psychometric function as a normal ogive) which has frequently been applied to temporal order judgment data (cf. Hirsh, 1959; Rutschmann, 1966).

\section{EXPERIMENT II}

This experiment examines the joint effect of preceding stimulus offset asychrony, and test stimulus onset asynchrony, on performance in an onset order judgment task. To specify these two independent variables more precisely, let the test onset asynchrony be denoted $A_{1}$, and defined by

$$
A_{1}=T_{L}-T_{R} \text {. }
$$

$T_{L}$ and $T_{R}$ are the (clock) onset times of the left and right test stimuli (Stimuli $\mathrm{C}$ and $\mathrm{D}$ in Figure 1a), respectively.

Similarly, let the preceding stimulus offset asynchrony be denoted $A_{2}$, defined by

$$
A_{2}=T_{L}^{*}-T_{R}^{*}
$$

$T_{L}^{*}$ and $T_{R}^{*}$ are the (clock) offset times of the left and right preceding stimuli (Stimuli $A$ and $B$ in Figure 1a), respectively. The main purpose of the experiment was to reveal the effects of $\mathrm{A}_{2}$ on the slope and location of the psychometric function, in an "IAE condition," in which $A_{1}$ and $A_{2}$ were varied factorially. A comparison between these functions and those obtained in a more conventional temporal order task (the "simple condition") is also described. The model is used to draw preliminary conclusions about the role of the IAE in temporal order judgments.

\footnotetext{
Method

Subjects. The author and two paid undergraduate assistants served as observers. ${ }^{1}$

Procedure. The display and control apparatus have been described for Experiment I. Details of the IAE condition will be described first. The spatial configuration of stimulus displays was as in Figure 1a. The sequence of events within a trial was as follows: (i) both preceding stimuli were illuminated for $1,000 \mathrm{msec}$; (ii) either light $A$ alone or light $B$ alone, or neither inducing light, remained illuminated for another $50 \mathrm{msec}$ (these three possibilities correspond respectively to $A_{2}=+50 \mathrm{msec}, A_{2}=-50 \mathrm{msec}$, and $A_{2}$ $=0 \mathrm{msec}$ ); (iii) an "all dark" interval of $50 \mathrm{msec}$ was presented; (iv) either light $C$ alone or light $D$ alone, or neither test light, was illuminated for $50 \mathrm{msec}$ (these three possibilities correspond, respectively to $A_{1}=-50 \mathrm{msec}, A_{1}=+50 \mathrm{msec}$, and $A_{1}=$ $0 \mathrm{msec}$ ); and (v) both test stimuli were illuminated together for 500 msec. These five time "frames" were immediately consecutive and nonoverlapping; thus the total duration of the stimulus pattern was a constant $1,650 \mathrm{msec}$. The central fixation point (see Figure 1a) remained illuminated throughout the five "frames." The "dark" interval between inducing stimulus offset and test stimulus
}

onset on either side (left or right) of the display could be 50,100 , or $150 \mathrm{msec}$, depending on the stimulus pattern defined by Frames ii and iv. The interval between "first offset" and "last onset," within which all of the manipulated stimulus information was presented, was a constant $150 \mathrm{msec}$. Both $A_{1}$ and $A_{2}$ assumed values of $-50,0$, and $+50 \mathrm{msec}$ with equal frequency, and values of the two variables were combined factorially to define nine different stimulus patterns. Each observer was seated in a testing chamber (Industrial Acoustics Corporation) and viewed the display unit through a viewing tunnel external to the window of the chamber. Viewing distance was about 30 in

Observers were asked to judge the "onset order", of the two test stimuli, indicating their response on each trial by pressing one of two buttons (designated "left first," an $R_{1}$ response, and "right first," an $R_{2}$ response). Trials were run in blocks of 135 , with short rest intervals between blocks. Within each block, the sequence of trials was random, with the constraint that each of the nine stimulus patterns occurred exactly 15 times. Typically, three or four blocks constituted one daily session for each observer. Three practice blocks and 11 data blocks were collected, giving 165 observations per stimulus pattern per observer.

Finally, all three observers were run in a "simple" temporal order task, with no inducing stimuli: that is, lights A and B in Figure 1a were never illuminated. The design and procedure of this simple condition, in which $A_{1}$ was the only independent variable, were comparable to the IAE condition, in which both $A_{1}$ and $A_{2}$ were manipulated. $A_{1}$ assumed values ranging from $-50 \mathrm{msec}$ to $+50 \mathrm{msec}$, in steps of $10 \mathrm{msec}$, randomly from trial to trial. The sequence of events within a trial was as follows: a fixation point located midway between the two test stimulus positions (corresponding to positions $C$ and D in Figure $1 \mathrm{a}$ ), was illuminated for $1,000 \mathrm{msec}$, followed by the illumination of the two test onsets with asynchrony $A_{1}$. The duration of the stimulus illuminated second was $500 \mathrm{msec}$. The durations of the response interval $(2,000 \mathrm{msec})$ and the intertrial interval $(1,000 \mathrm{msec})$ were the same as in the IAE condition. Within each 165-trial block, each of the 11 values of $A_{1}$ occurred 15 times. Three practice and nine data blocks were collected, giving 135 observations per stimulus pattern per observer.

\section{Results}

For the IAE condition, the proportions of "right-first" responses conditional on each combination of $A_{1}$ and $A_{2}$ values are entered in Table 2 for each observer. Both independent variables exert pronounced effects on response tendency. The direction of the effect due to manipulating $A_{2}$ is consistent with the results of Experiment $I$.

For the simple condition, the proportions of "right-first" responses conditional on each value of A are entered in Table 3 for each observer.

Theoretical analysis. Considering first the IAE condition, Figure 2 shows for each observer a plot of $\hat{Z}\left(R_{2} \mid A_{1}\right)$, with $A_{2}$ as parameter. For each value of $A_{2}$, a linear psychometric function has been fit to the data. These three functions differ in location but not in slope for each observer, and appear to conform reasonably well with the pattern of data. ${ }^{2}$ The predicted response probabilities are given in Table 2 . A chi-square comparison of observed and predicted response frequencies for the pooled data of all three observers yielded a value of 3.53 , which does not approach significance ( $p>.05, \mathrm{df}=5$ ). By contrast, functions which differ in slope but not in location are clearly not appropriate, while functions which differ 
Table 2

Observed $\hat{\mathbf{P}}\left(\mathbf{R}_{2} \mid \mathbf{A}_{1}\right)$, Predicted $\mathbf{P}\left(\mathbf{R}_{2} \mid \mathbf{A}_{1}\right)$ in Parintheses, and Estimates of $a_{m}$, for Each Value of $A_{2}$, and of $\sigma_{X}$, for Each Observer, in the IAE Condition: Experiment II

\begin{tabular}{|c|c|c|c|c|c|c|}
\hline \multirow{2}{*}{$\overline{A_{1}=-50 \mathrm{msec}}$} & \multicolumn{6}{|c|}{ Observer } \\
\hline & \multicolumn{2}{|c|}{ C.C. } & \multicolumn{2}{|c|}{ R.H. } & \multicolumn{2}{|c|}{ J.W. } \\
\hline $\begin{array}{l}A_{2}=-50 \\
A_{2}=0 \\
A_{2}=+50\end{array}$ & $\begin{array}{l}.01 \\
.04 \\
.15\end{array}$ & $\begin{array}{l}(.01) \\
(.05) \\
(.09)\end{array}$ & $\begin{array}{l}.08 \\
.14 \\
.20\end{array}$ & $\begin{array}{l}(.07) \\
(.12) \\
(.20)\end{array}$ & $\begin{array}{l}.33 \\
.37 \\
.50\end{array}$ & $\begin{array}{l}(.29) \\
(.37) \\
(.51)\end{array}$ \\
\hline $\begin{aligned} \mathrm{A}_{1}=0 \mathrm{msec} \\
\mathrm{A}_{2}=-50 \\
\mathrm{~A}_{2}=0 \\
\mathrm{~A}_{2}=+50\end{aligned}$ & $\begin{array}{l}.19 \\
.42 \\
.51\end{array}$ & $\begin{array}{l}(.16) \\
(.37) \\
(.49)\end{array}$ & $\begin{array}{l}.23 \\
.32 \\
.39\end{array}$ & $\begin{array}{l}(.23) \\
(.34) \\
(.45)\end{array}$ & $\begin{array}{l}.36 \\
.47 \\
.61\end{array}$ & $\begin{array}{l}(.41) \\
(.50) \\
(.63)\end{array}$ \\
\hline $\begin{array}{c}\mathrm{A}_{1}=+50 \mathrm{msec} \\
\mathrm{A}_{2}=-50 \\
\mathrm{~A}_{2}=0 \\
\mathrm{~A}_{2}=+50\end{array}$ & $\begin{array}{l}.68 \\
.84 \\
.84\end{array}$ & $\begin{array}{l}(.63) \\
(.83) \\
(.90)\end{array}$ & $\begin{array}{l}.48 \\
.63 \\
.79\end{array}$ & $\begin{array}{l}(.50) \\
(.63) \\
(.74)\end{array}$ & $\begin{array}{l}.54 \\
.64 \\
.77\end{array}$ & $\begin{array}{l}(.54) \\
(.62) \\
(.75)\end{array}$ \\
\hline $\begin{array}{l}\hat{a}_{m} \mid A_{2}=-50 \\
\hat{a}_{m} \mid A_{2}=0 \\
\dot{a}_{m} \mid A_{2}=+50 \\
\hat{\sigma}_{X} \\
\text { Proportion* }\end{array}$ & $\begin{array}{l}37 \\
12 \\
38 \\
\end{array}$ & 83 & $\begin{array}{r}50 \\
27 \\
7 \\
67\end{array}$ & 82 & $\begin{array}{r}35 \\
2 \\
-52 \\
153\end{array}$ & 60 \\
\hline
\end{tabular}

*Proportion of variance accounted for.

in slope as well as location seemed not to improve the tit of the model enough to warrant the estimation of six, rather than four, theoretical parameters for each observer. Hence, in terms of the model, it is reasonable to attribute the effect of $A_{2}$ in this experiment, solely to changes in the value of $\mathrm{a}_{\mathrm{m}}$. The value of $\hat{\sigma}_{\mathrm{X}}$ and three values of $\hat{a}_{m}$ (one for each value of $A_{2}$ ) are entered in Table 2 for each observer, together with the proportion of data variance accounted for by the model. Note that the present data are not sufficient to determine the relative contributions of $u_{P}$ and $u_{C}$ to the IAE.

Regarding the simple condition, predicted response probabilities were obtained from the linear function which best tit the values of $\hat{Z}\left(R_{2} \mid A_{1}\right)$ for each observer. These predictions. together with the proportions of data variance accounted for by the model, and estimates of $a_{m}$ and $\sigma_{X}$ for each observer, are included in Table 3 . The fit of the model is adequate for observers C.C. and R.H., but relatively poor for observer J.W., whose data depart from monotonicity as well as from linearity for positive values of $\mathrm{A}_{1} \cdot{ }^{3}$ The validity of parameter estimates based on the latter set of data is therefore questionable. However, if anything. it seems that the linear function tit to observer J.S.'s data provides an underestimation of his sensitivity, since he does appreciably better on negative values of $A_{1}$. Thus one can argue in general that all three observers showed higher discrimination in the simple condition than in the IAE condition.

The lower slopes (higher values of $\dot{u}_{\mathrm{X}}$ ) in the IAE condition suggest that additional "noise" is present when the inducing stimuli are added to the stimulus pattern. One interpretation of this result is that the inducing stimuli in the IAE condition produce lateral masking, which degrades the sensory registration of the test onsets. Another interpretation is that observers respond, to some extent, on the basis of $A_{2}$. and, to that extent, independently of $A_{1}$. Two versions of this second hypothesis are: (i) that on some proportion of trials the observer's response is "cued" or determined solely by the offset order of the inducing stimuli; and (ii) that the observer responds on the basis of a weighted combination of internal effects associated with both $A_{1}$ and $A_{2}$ on every trial. Both versions of this hypothesis predict that: (i) the slope of the psychometric function will be lower in the IAE condition than in the simple condition; and (ii) that the slope of the psychometric function will not be independent of $A_{2}$ if some values of $A_{2}$ exert more control over the observer's response than other values. The lower slopes (higher values of $\hat{\sigma}_{X}$ ) in the IAE condition are consistent with the first prediction. For most of the data, slopes that are independent of $\mathrm{A}_{2}$ give a good account of the experimental results. ${ }^{4}$ This is another way of saying that the effects of $A_{1}$ and $A_{2}$ are additive; hence, one can draw the important conclusion that observers utilize the information available about $A_{1}$ to about the same extent over all values of $A_{2}$. For example, observers do not appear to base their responses on the stronger of the two internal effects associated with $A_{1}$ and $A_{2}$ on each trial.

It is interesting to note that the results of Experiment II are inconsistent with the hypothesis that the observer bases his response on the relative durations of the intervals between inducing stimulus oftset and test stimulus onset on each side of the display. Since the duration of the interstimulus interval between right offset and onset is a decreasing function of $A_{1}$, one strategy available to the observer is

Table 3

Observed $\hat{\mathbf{P}}\left(\mathbf{R}_{2} \mid \mathbf{A}_{1}\right)$, Predicted $\mathbf{P}\left(\mathbf{R}_{2} \mid \mathbf{A}_{1}\right)$ in Parentheses, and Parameter Estimates for Each Observer in the Simple Condition: Experiment II

\begin{tabular}{|c|c|c|c|c|c|c|}
\hline & \multicolumn{6}{|c|}{ Observer } \\
\hline & \multicolumn{2}{|c|}{ C.C. } & \multicolumn{2}{|c|}{ R.H. } & \multicolumn{2}{|c|}{ J.W. } \\
\hline \multicolumn{7}{|l|}{ A msec } \\
\hline-50 & .01 & $(.01)$ & .10 & $(.05)$ & .13 & $(.24)$ \\
\hline-40 & .02 & $(.03)$ & .07 & $(.09)$ & .25 & $(.29)$ \\
\hline-30 & .05 & $(.06)$ & .13 & $(.14)$ & .39 & $(.35)$ \\
\hline-20 & .08 & $(.12)$ & .19 & $(.22)$ & .46 & $(.40)$ \\
\hline-10 & .26 & $(.21)$ & .28 & $(.31)$ & .54 & $(.46)$ \\
\hline 0 & .40 & $(.34)$ & .38 & $(.42)$ & .67 & $(.52)$ \\
\hline 10 & .55 & $(.48)$ & .46 & $(.54)$ & .59 & $(.58)$ \\
\hline 20 & .59 & $(.64)$ & .62 & $(.65)$ & .70 & $(.64)$ \\
\hline 30 & .79 & $(.77)$ & .75 & $(.75)$ & .61 & $(.69)$ \\
\hline 40 & .86 & $(.87)$ & .89 & $(.83)$ & .68 & $(.74)$ \\
\hline 50 & .90 & $(.93)$ & .91 & $(.89)$ & .74 & $(.79)$ \\
\hline$\hat{\sigma}_{\mathbf{X}}$ & \multicolumn{2}{|c|}{26.3} & \multicolumn{2}{|c|}{34.5} & \multicolumn{2}{|c|}{66.7} \\
\hline$\hat{a}_{m}$ & \multicolumn{2}{|c|}{+10.9} & \multicolumn{2}{|c|}{+7.0} & \multicolumn{2}{|c|}{-3.2} \\
\hline Proportion* & \multicolumn{2}{|c|}{.986} & \multicolumn{2}{|c|}{.982} & \multicolumn{2}{|c|}{.835} \\
\hline
\end{tabular}

*Proportion of variance accounted for. 

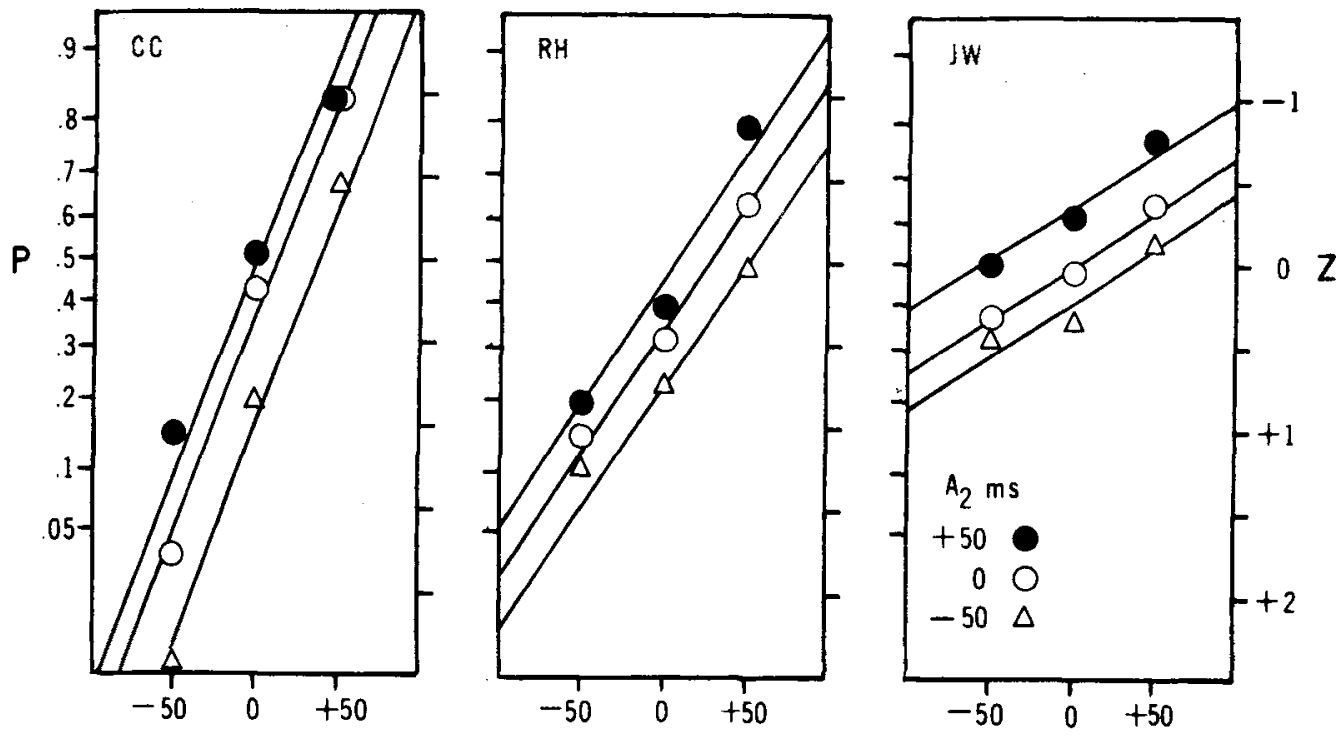

$A_{1} \mathrm{~ms}$

Flgure 2. Results of Experiment II, for three observers. Data points represent observed proportions of "right-onset-first" responses. Linear functions show the model's predicted pattern of results. The ordinate is a Gaussian-transformed probability scale (probit scale).

to respond "right first" $\left(\mathbf{R}_{2}\right)$ when the right offset-onset interval appears shorter in duration than the left interval, and to respond "left first" $\left(R_{1}\right)$ otherwise. The direction of the effect of $A_{2}$ is inconsistent with the hypothesis that observers use such a strategy. This is so because the duration of the right interstimulus interval is an increasing function of $A_{2}$. Thus, under the duration strategy hypothesis, the proportion of $R_{2}$ responses is predicted to be a decreasing function of $A_{2}$. The data show that the proportion of $R_{2}$ responses is an increasing function of $A_{2}$. Therefore, the hypothesis predicts that the direction of the effect of $A_{2}$ should be the opposite of the direction observed. A similar argument rules out the hypothesis that observers base their judgments on the relative velocities of apparent motion on each side of the display.

To summarize, Experiment II, together with the foregoing analysis, (i) provides a demonstration of the IAE; (ii) shows that the model gives one reasonable and simple account of the pattern of data, in which the effects of $A_{1}$ and $A_{2}$ are, to a close approximation, additive with respect to a Gaussian transformation; (iii) shows that the slope of the psychometric functions in the IAE condition differs systematically from the slope of the comparable function for simple temporal order discrimination; (iv) advances two types of explanation for this difference; and (v) shows that two plausible hypotheses concerning the basis of the observers' judgments are inconsistent with the direction of the effect of $A_{2}$.

\section{DISCUSSION}

In developing the model in its present form, it was emphasized that changes in the location of the psychometric function are problematic, because $u_{C}$ and $u_{P}$ cannot be separately estimated from temporal order data alone. The IAE is an example of this problem. Further research will be necessary in order to partial out the relative contributions of perceptual error and response bias to the value of $a_{m}$.

One can speculate about the potential theoretical resolutions of this problem in the case of the IAE. Perhaps the most exciting possibility is that the variable $A_{2}$ controls rapid shifts in the distribution of attention over the visual field. If, as several investigators (e.g., Sternberg \& Knoll, 1973) maintain, the arrival latency of attended stimuli is shorter than that of nonattended stimuli, then, under the attention hypothesis, the IAE would be a "prior entry" effect under very good stimulus control. Furthermore, performance in information-processing tasks other than temporal order might be influenced by similar induction manipulations. It would be interesting to know, for example, whether $\mathbf{A}_{2}$ influences the accuracy of recognition of letters presented briefly in the spatial vicinity of the inducing stimuli.

A second possibility is that $A_{2}$ influences the value of $u_{P}$, not as a consequence of shifts in attention, but more directly, and without effects on other kinds of processing. Third, the IAE may be "simply" a 


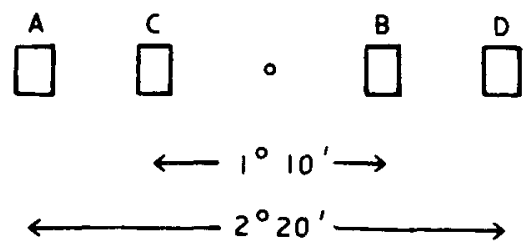

(a)

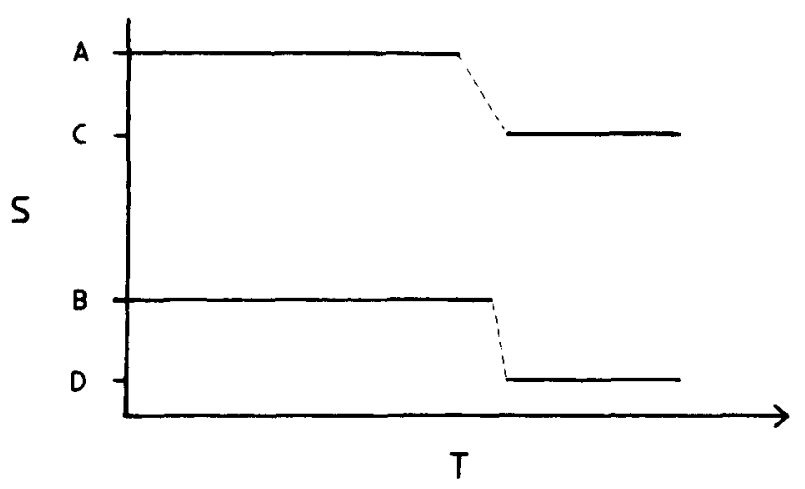

(b)

Figure 3. (a) Linear array version of IAE display. (b) Representation of an IAE trial using linear array. Solid lines denote the on periods of stimuli in spatial positions given by ordinate; dashed lines represent apparent motion between successive stimuli.

response bias effect, reflecting changes in the value of $\mathrm{u}_{\mathrm{c}}$ alone. Finally, there may be some contribution to the effect from both perceptual error and response bias. The existence of these diverse and interesting possibilities should encourage the development of new experiments and theory.

Many problems of current interest in perception (e.g. . tachistoscopic letter recognition, visual masking, apparent motion, temporal order, and duration discrimination) are investigated by requiring observers to extract from a stimulus pattern, within a very brief interval of time, information which is sufficient to discriminate one pattern from another. Kahneman (1968) has made a related observation in discussing various limiting cases of visual masking paradigms. Investigators in a number of problem areas, then, are interested in discovering general principles governing the perception of rapidly changing visual displays.

The IAE is a relatively simple dynamic phenomenon which may be useful in discovering such principles. Although a detailed parametric analysis has not yet been carried out, inspection of a few variants of the effect has revealed interesting similarities between the IAE and three other dynamic perceptual phenomena. The following discussion develops these similarities and suggests one way of conceptualizing them.
An induced asynchrony effect can be obtained using the configuration of stimulus positions shown in Figure 3a. The time course of their presentation is again illustrated by Figure 1b. As before, the onset of that test stimulus closest to the first preceding stimulus to be extinguished tends to be judged earlier than the other test onset. Figure $3 b$ is a "space-time diagram" representing this dynamic stimulus pattern. In this diagram, the ordinate represents spatial positions along the horizontal dimension of the stimulus array; the abscissa is time. Thus, the velocity of apparent motion between, for example, pósitions A and $C$ is represented by the slope of the dashed line connecting the offset at $\mathrm{A}$ and the onset at $\mathrm{C}$. Note that the IAE, which in the example of Figure $1 \mathrm{~b}$ is a tendency to judge the onset at $\mathrm{C}$ to be earlier than the onset at $D$, would have the effect of forcing the velocities of apparent motion toward equality.

A simpler example is shown in Figure 4a, which represents a three-stimulus array. The temporal pattern of illumination is as follows: one of the end lights (A) is presented briefly, followed after a brief dark interval by the simultaneous presentation of the middle light $(B)$ and the other end light $(C)$. Observers

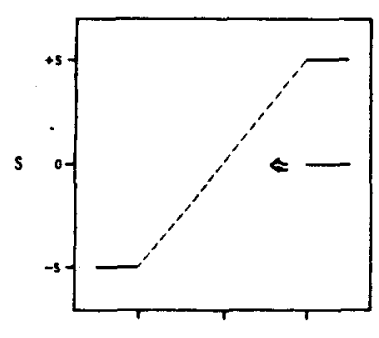

(a)

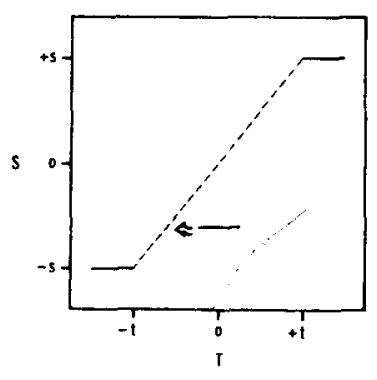

(c)

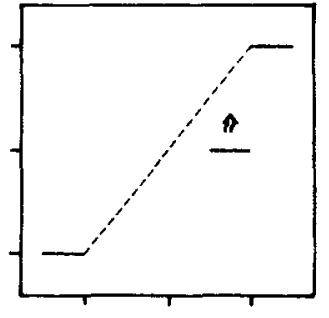

(b)

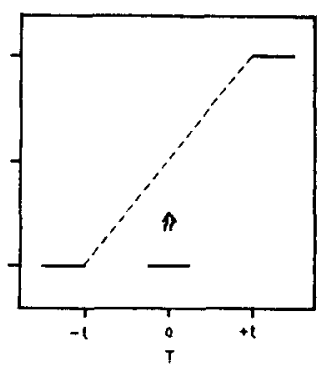

(d)
Figure 4. Space-time diagrams representing four dynamic perceptual "illusions": (a) simple form of IAE, (b) tau effect, (c) kappa effect, (d) simple form of rabbit effect. The paradigms are arranged in the figure as follows: Top row, "even" spacing and "uneven" timing; bottom row, "uneven" spacing and "even" timing; left column, temporal judgment tasks; right column, spatial judgment tasks. Arrows represent the direction of perceptual distortion reported for each paradigm. The observer may have a tendency to judge the spatial positions or times of presentation of the three stimuli in such a way as to preserve constant velocity of apparent motion, which is represented by the dashed line. 
viewing this pattern tend to judge the onset of $B$ to be earlier than the onset of $C$. (The phenomenal impression is weaker than in the original IAE configuration; however, this simple pattern may be the IAE's minimal form) Again, the effect is in the direction of minimizing the difference in slope between the apparent motions A-to-B and A-to-C.

Three other effects in the literature (which have been studied cutaneously as well as visually) are also represented in Figure 4. The tau effect (Helson \& King, 1931) and the rabbit effect (Geldard \& Sherrick, 1972) are spatial distortions in the judged position of the second of three stimuli. The kappa effect (Cohen, Hansel, \& Sylvester, '1955), like the IAE, is a temporal distortion. The tau and Kappa effects have, at various times, caught the imagination of workers in perception because these phenomena demonstrate interdependence between psychological space and time, the main dimensions of our sensory experience. Although it has been recognized (Cohen, 1967) that the tau and kappa paradigms are, in a way, "mirror images" of each other, an attempt to classify spatio-temporal "illusions" did not appear to be conceptually promising until recently, with the discovery of the "rabbit," and now the IAE. Figure 4 represents an attempt by the author to organize the essential similarities and differences among these four phenomena. The figure caption summarizes this organization.

The simple IAE and the tau effect have been observed in stimulus patterns where the two spatial intervals separating the three stimuli are equal, but the temporal intervals are unequal. The rabbit and kappa effects have been observed under conditions of equal time intervals but unequal spatial intervals. In all four phenomena, the perceptual distortion is in the direction that would be expected under the hypothesis that observers have a tendency toward constant velocity in the perception of apparent motion. For example, the tau effect in Figure $4 \mathrm{~b}$ is a distortion in the judged position of the middle stimulus, which tends to be displaced in psychological space toward the third stimulus, which is closest to it in time.

It may be profitable for future investigators to consider these four paradigms together, and to generalize them; until now, research efforts have tended to focus on one or another of the effects. Some unanswered questions, for example, are: (i) Can all four phenomena be obtained over the same ranges of space and time? (ii) What is their relationship to other information-processing problems, such as masking? (iii) Is the observer's task (time or space judgment) the only difference between tau and kappa under conditions in which both the spacing and the timing of the three stimuli are uneven?

\section{REFERENCES}

BARON, J. Temporal ROC curves and the psychological moment. Psychonomic Science, 1969, 15, 299-300.

Conen. J. Psychological time in health and disease. Springfield, III: Thomas, 1967.

Cohen, J., Hansel, C. E. M., \& Sylvester, J. D. Interdependence in judgments of space, time and movement. Acta Psychologia, 1955, 11, 360-372.

Geldard, F. A., \& Sherrick, C. E. The cutaneous "rabbit": A perceptual illusion. Science, 1972, 178, 178-179.

Gibmon, J.. \& Rutschmann, R. Temporal order judgment and reaction time. Science, $1969,165,413-415$.

Helson, H., \& King, S. M. The tau effect: An example of psychological relativity. Journal of Experimental Psychology, 1931. 14. 202-217.

HIRSH, I. J. Auditory perception of temporal order. Journal of the Acoustical Society of A merica, 1959, 31, 759-767.

KaHNEMAN, D. Method, findings and theory in studies of visual masking. Psychological Bulletin, 1968, 69, 404-425.

Komoda, M. K., Festinger, L., Phillips, L. J., Duckman, R. H., \& Young, R. A. Some observations concerning saccadic eye movements. Vision Research, 1973, 13, 1009-1020.

Rutschmann, R. Perception of temporal order and relative visual latency. Science, 1966, 152, 1099-1101.

Sternaerg, S., \& KNOLL, R. L. The perception of temporal order: Fundamental issues and a general model. In S. Kornblum (Ed.), Attention and performance IV. New York: Academic Press, 1973.

\section{NOTES}

1. One observer, J.W., had previously been a subject in Experiment 1, Group II; his judgment of Sequence $R$ was "left first," a reversal of the IAE. That this one response does not reflect a basic difference between J.W. and most other observers is shown by the results of Experiment II. in which all three observers, over many trials, displayed the IAE in a consistent way.

2 . The slope (from which an estimate of $\sigma X$ can be derived) was obtained by applying simple regression analysis to the averages of the three $Z$ values associated with each value of $A_{1}$. The $\hat{Z}$ intercepts (from which estimates of the corresponding values of $a_{m}$ can be derived) were obtained by averaging the three $\hat{Z}$ values associated with each value of $A_{2}$.

3. A chi-square comparison of observed and predicted response frequencies in the simple condition yielded the following results. Obtained values of 12.16 and 16.16 for observers C.C. and R.H., respectively, indicated no significant differences $(\mathrm{p}>.05, \mathrm{df}=9$ ). An obtained value of 40.52 for observer J.W. was significant $(\mathrm{p}<.005$ ), indicating poor correspondence with the model for this set of data.

4. Chi-square comparisons between observed and predicted response frequencies were carried out for each observer's data. The data of observers R.H. and J.W., with obtained values of 5.70 and 4.65, respectively, showed no significant departure from the predictions of the model $(\mathrm{p}>.05, \mathrm{df}=5$ ). However, a value of 19.25 was obtained for C.C.'s data, which represents a significant difference. Most $(68 \%)$ of this difference is attributable to the data for $A_{2}=+50$, which displays a slightly lower slope than predicted. As indicated in the text, one interpretation of such a slope decrement is that there is a slightly higher tendency to respond on the basis of $A_{2}$ for this value of $A_{2}$.

(Received for publication May 7, 1975; revision received September 23, 1975.) 